\title{
Zero-mode contribution and quantized first-order apparent phase transition in a droplet quark matter
}

\author{
Kun $\mathrm{Xu}^{1,2, \dagger}$ and Mei Huang ${ }^{1, *}$ \\ ${ }^{1}$ School of Nuclear Science and Technology, University of Chinese Academy of Sciences, \\ Beijing 100049, China \\ ${ }^{2}$ Institute of High Energy Physics, Chinese Academy of Sciences, \\ Beijing 100049, People's Republic of China
}

(Received 6 June 2019; revised manuscript received 18 December 2019; accepted 7 March 2020; published 2 April 2020)

\begin{abstract}
The finite size effect on hadron physics and quark matter has attracted much interest for more than three decades; normally, both the periodic (with a zero-momentum mode) and the antiperiodic (without a zeromomentum mode) spatial boundary condition are applied for fermions. By comparing the thermodynamical potential, it is found that, if there is no other physical constraint, the droplet quark matter is always more stable when the periodic spatial boundary condition is applied, and the catalysis of chiral symmetry breaking is observed with the decrease of the system size, while the pions excited from the droplet vacuum remain as pseudo-Nambu-Goldstone bosons. Furthermore, it is found that the zero-momentum-mode contribution brings a significant change of the chiral apparent phase transition in a droplet of cold dense quark matter: The first-order chiral apparent phase transition becomes quantized; i.e., the first-order apparent phase transition is completed in two steps, which is a brand-new quantum phenomenon. It is expected that the catalysis of chiral symmetry breaking and the quantized first-order phase transition are common features for fermionic systems with a quantized momentum spectrum with a zero-mode contribution, which also shows up in quark matter under a magnetic field.
\end{abstract}

DOI: 10.1103/PhysRevD.101.074001

\section{INTRODUCTION}

The size effect attracts wide interest in different physical systems. For example, in a recent article [1], scientists realized that the most essential factor of making a grape plasma in a microwave oven is the grape size, which is comparable with the typical microwave length, so that the grape can "trap" microwaves. Finite size effects on phase transitions were studied four decades ago [2], and the finite size effect in quantum chromodynamics (QCD) of hadron physics has attracted much interest for more than three decades [3-7], which is important to extract hadron properties from numerical simulations on finite and discrete Euclidean space-time lattices. The study of the finite size effect on quark matter and QCD phase structure becomes necessary and important [8-14] due to the fact that the hot and dense matter created through heavy-ion collisions has a

\footnotetext{
* Corresponding author.

huangmei@ucas.ac.cn

†xukun@mail.ihep.ac.cn

Published by the American Physical Society under the terms of the Creative Commons Attribution 4.0 International license. Further distribution of this work must maintain attribution to the author(s) and the published article's title, journal citation, and DOI. Funded by SCOAP.
}

finite volume with the typical size of a nuclei. The quark droplet has also been investigated in neutron stars [15]. When considering the mixed phase of quark matter and nuclear matter, one has to solve the Wigner-Seitz cell structure, e.g., drop (bubble), rod (tube), and slab structure, and it is found that the size of the Wigner-Seitz cell can be as small as several femtometers as shown in Ref. [16].

As there is a typical length in grape plasma, i.e., the microwave length, in a QCD system the typical length is the pion Compton length $\lambda_{\pi}=1 / m_{\pi} \sim 1.41 \mathrm{fm}$. When the system size is much larger than the pion Compton length $L \gg \lambda_{\pi}$, hadron properties and phase transitions satisfy some finite size scaling behavior [2,5]. When the system size is comparable to or even smaller than the pion Compton length $L \sim \lambda_{\pi}$, the size effect becomes significant.

In the case of finite volume, the general method is to replace the momentum integral to momentum summation, i.e., $\int \frac{d^{3} p}{(2 \pi)^{3}} \rightarrow \frac{1}{V} \sum_{p}$, with $V$ the volume of the system. For bosons, the most natural choice of boundary condition in the spatial direction is periodic, which implies that the momentum is summed from the exact zero-momentum mode $\vec{p}=0$ in a finite system. However, for fermions or quarks in a finite system, its boundary condition (BC) in the spatial directions is not determined by a physical constraint, and one is free to choose either periodic $(\mathrm{P})$ or antiperiodic (AP) boundary conditions. In lattice QCD simulations, the 
periodic boundary condition (PBC) is normally applied for fermions or quarks, and the antiperiodic boundary condition (APBC) is applied in most cases for fermions in the spatial direction in order to keep the so-called permutation symmetry between the time and space directions $[13,14]$ and also to be consistent with the results of the volumedependent pion mass from the chiral perturbation theory [5].

The boundary condition becomes important when the system size is comparable to the pion wavelength. Applying the APBC and PBC to fermions induces two opposite results on the properties of the QCD vacuum: The APBC induces chiral symmetry restoration, while the PBC induces the catalysis of chiral symmetry breaking in the vacuum. The catalysis of chiral symmetry breaking in a small system with a PBC including the zero-momentummode contribution immediately reminds us of the system of quark matter under a strong magnetic field $B[17,18]$, where the summation of discrete energy is taken from the lowest Landau level, which is basically the zeromomentum mode. It is not difficult to understand the similarity between the two systems, if we recognize that, for a charged particle carrying charge $q$, the magnetic length $l$ is proportional to the inverse of the square root of the magnetic field, i.e., $l \sim \frac{1}{\sqrt{|q| B}}[19]$; thus, the stronger the magnetic field, the smaller the magnetic length will be.

In this work, we carefully investigate quark matter in a finite system with both the antiperiodic and periodic spatial boundary conditions applied for quarks and analyze the two different physical results. In Sec. II, we compare the thermodynamical potential of the small size system applying the PBC (with a zero mode) and APBC (without a zero mode), respectively, and the lower thermodynamical potential determines the stable ground state of the small system. The results of catalysis of chiral symmetry breaking and pseudo-Nambu-Goldstone (NG) pions are obtained in Sec. III, and then in Sec. IV we show the quantized firstorder phase transition in cold droplet quark matter. Last, we give a summary and discussion. It is worth mentioning that actual phase transitions are possible only for infinite volumes; thus, we use "apparent phase transition" for finite size systems in the whole paper.

\section{DROPLET QUARK MATTER WITH THE PBC AND APBC FOR QUARKS}

It is noticed that, in this work, we focus only on discussing the boundary condition of fermions and neglect the finite size effect from gluon dynamics; therefore, we can use the simplest four-fermion interacting Nambu-JonaLasinio (NJL) model. The Lagrangian density of the twoflavor NJL model with an interaction only in the scalar channel [20] is given by

$$
\mathcal{L}=\bar{\psi}\left(i \gamma^{\mu} \partial_{\mu}-m\right) \psi+G\left[(\bar{\psi} \psi)^{2}+\left(\bar{\psi} i \gamma^{5} \vec{\tau} \psi\right)^{2}\right],
$$

where $\psi=(u, d)^{T}$ is the doublet of the two light quark flavors $u$ and $d$ with the current mass $m=m_{u}=m_{d}$ and $\vec{\tau}=\left(\tau^{1}, \tau^{2}, \tau^{3}\right)$ the isospin Pauli matrix. Introducing the auxiliary scalar and pseudoscalar fields $\sigma=-2 G\langle\bar{\psi} \psi\rangle$ and $\vec{\pi}=-2 G\left\langle\bar{\psi} i \gamma_{5} \vec{\tau} \psi\right\rangle$ and considering only the scalar condensation in the vacuum, the effective potential of the system in the Pauli-Villars regularization scheme takes the form of

$$
\begin{aligned}
\Omega= & \frac{\sigma^{2}}{4 G}-2 N_{c} N_{f} \int_{-\infty}^{\infty} \frac{d^{3} p}{(2 \pi)^{3}}\left[\sum_{j=0}^{3} c_{j} \sqrt{E^{2}+j \Lambda^{2}}\right. \\
& \left.+T \ln \left(1+e^{-[(E+\mu) / T]}\right)+T \ln \left(1+e^{-[(E-\mu) / T]}\right)\right],
\end{aligned}
$$

with $M=m+\sigma$ the constituent quark mass, the dispersion relation $E=\sqrt{p^{2}+M^{2}}, T$ the temperature, and $\mu$ the quark chemical potential, respectively. Model parameters are fixed as $c_{0}=1, c_{1}=-3, c_{2}=3, c_{3}=-1$, and $\Lambda=$ 782.37 MeV and $G=6.197 / \Lambda^{2}$ by fitting pion decay constant $f_{\pi}=93 \mathrm{MeV}$ and quark constitute mass $M=$ $330 \mathrm{MeV}$.

Putting quark matter in a cubic box with finite length $L$, the momentum integral is replaced by the summation of the discrete momentum $\int \frac{d^{3} \vec{p}}{(2 \pi)^{3}} \rightarrow \frac{1}{V} \sum_{\vec{p}}$ with $V=L^{3}$ the volume of the system. There is no strict rule to rule out either $\mathrm{PBC} \quad \vec{p}^{2}=\left(\frac{2 \pi}{L}\right)^{2} \sum_{i=x, y, z} n_{i}^{2} \quad$ or $\mathrm{APBC} \quad \vec{p}^{2}=$ $\left(\frac{2 \pi}{L}\right)^{2} \sum_{i=x, y, z}\left(n_{i}+\frac{1}{2}\right)^{2}$ with $n_{i}=0,1,2, \ldots$ for fermion momentum summation; therefore, both spatial boundary conditions have been applied for fermions for several decades in the literature [8-14].

It is well known that the ground state of the system is determined by the effective potential. We show the effective potential of the finite size system by applying the PBC and APBC in Fig. 1. At $T=0, \mu=0$, it is observed that, when applying the PBC for quarks, the effective potential becomes lower with a decreasing size, while when applying the APBC for quarks, the effective potential becomes higher with a decreasing size. This indicates that, if there is no other physical constraint, the quark droplet prefers the PBC for quarks, in which the zero-momentum mode is taken into account.

From the effective potential in Fig. 1, we can read the chiral condensation in the vacuum, and the corresponding constituent quark mass and pion mass are shown in Fig. 2 by applying the $\mathrm{PBC}$ and $\mathrm{APBC}$ for quarks, respectively. We can read that when applying the PBC for quarks, with the decreasing of the system size, the chiral condensate enhances while the pion mass remains a constant. This is the familiar phenomenon of catalysis of chiral symmetry breaking also observed in quark matter under strong magnetic fields, where only the neutral pion remains a pseudo-NG boson. On the other hand, if the APBC is 


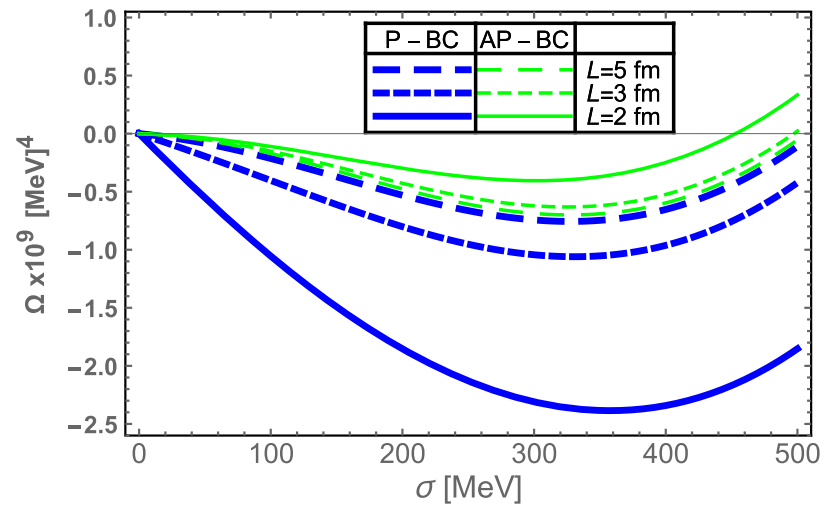

FIG. 1. The effective potential of the small system for three different sizes $L=5,3,2 \mathrm{fm}$ as a function of the chiral condensate $\sigma$ at $T=0, \mu=0$ by applying the periodic (blue lines) and antiperiodic (green lines) spatial boundary conditions for quarks, respectively.

applied for quarks, it is found that the chiral symmetry is restored in a small system and pions become heavier in the vacuum.

The main difference between the $\mathrm{PBC}$ and $\mathrm{APBC}$ is whether to take into account the zero-momentum-mode contribution. For discrete momentum, the gap between the zero mode and the first mode is $\frac{2 \pi}{L}$; therefore, when the system becomes small enough, the zero-momentum-mode contribution becomes dominant, which can be seen from the constituent quark mass with only a zero-mode contribution, as shown in Fig. 2, where $M_{0}$ and $M_{1}$ are obtained by solving gap equation (4) with and without a zero-mode contribution, respectively. This is also the same as the case of a strong magnetic field where the lowest Landau-level contribution dominates. Remember that, for a charged particle carrying charge $q$, the magnetic length $l$ is proportional to the inverse of the square root of magnetic field, i.e., $l \sim \frac{1}{\sqrt{|q| B}}$; thus, putting charged particles under

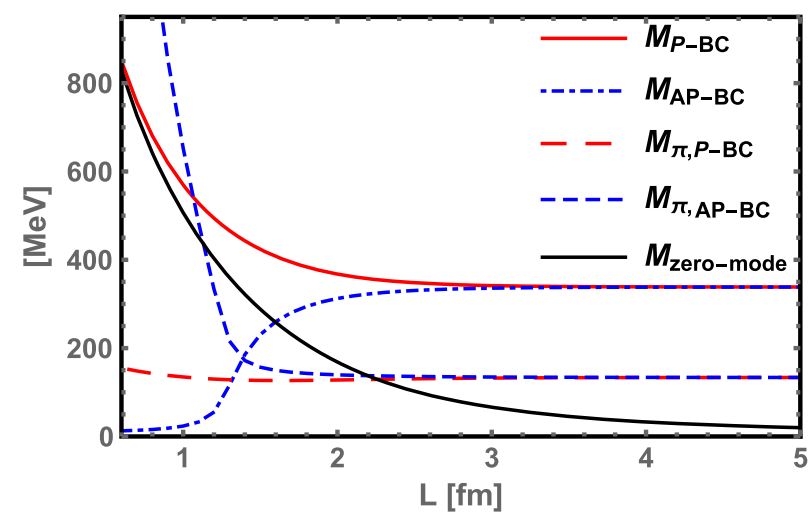

FIG. 2. The constituent quark mass and pion mass as a function of the system size $L$ at $T=0, \mu=0$ by applying the PBC and APBC for quarks, respectively. $M_{\text {zero-mode }}$ is obtained with only a zero-mode contribution.

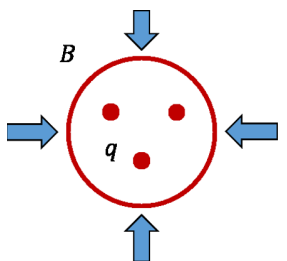

(a) MIT bag model

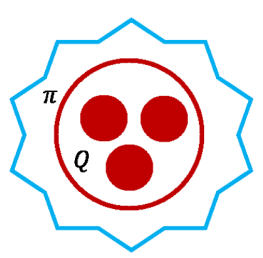

(b) Quark droplet with PBC

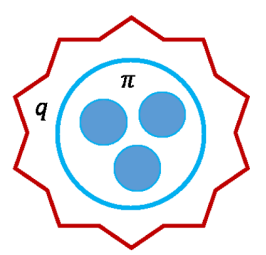

(c) Quark droplet with APBC
FIG. 3. The schematic picture for three scenarios of droplet quark matter: (a) The MIT bag model consists of free quarks $q$ with the bag constant $B$ putting by hand at the surface of the bag. (b) The quark droplet with a periodic boundary condition consists of massive quarks $Q$ with catalysis of chiral symmetry breaking and the pseudo-Nambu-Goldstone pions' cloud. (c) The quark droplet with an antiperiodic boundary condition consists of heavy pions inside the bag and light quarks' cloud. It is noticed that here the numbers and colors of quarks are shown in a schematic way.

the strong magnetic field in some sense is similar to putting these particles in an elongated cylinder with small radius $l$.

Furthermore, it is very interesting to notice that the difference of the thermodynamical potential or the pressure difference between $L=2 \mathrm{fm}$ and $L=\infty$ is on the order of $[200 \mathrm{MeV}]^{4}$, which is the typical value of the bag constant in the MIT bag model [21]. If we consider the spherical shape droplet quark matter, we will obtain qualitatively the same results as that for cubic droplet quark matter shown in Figs. 1 and 2 when the PBC and APBC are applied for quarks, respectively. In Fig. 3, we show three scenarios of a quark droplet: (a) MIT bag model, (b) quark droplet with the PBC, and (c) quark droplet with the APBC. The MIT bag model consists of free quarks $q$ with the bag constant $B$ putting by hand at the surface of the bag, and the quark droplet with the PBC consists of massive quarks $Q$ with catalysis of chiral symmetry breaking and the pseudoNambu-Goldstone pion cloud. However, for the quark droplet with the APBC, the quarks restore chiral symmetry and the quark mass becomes very small; instead, the pions become very heavy, and, therefore, we can imagine that the heavy pions stay inside the bag with a light quark cloud. We can see that the quark droplet with the PBC is very similar to the scenario of the MIT bag model, except that we can have spontaneous chiral symmetry breaking as well as Nambu-Goldstone pions in the quark droplet with the PBC. Therefore, the quark droplet with the PBC looks like a bag model with dynamical quarks; on the other hand, the quark droplet with the APBC is a bag with heavy pions. Of course, in this paper, we are not intending to derive the MIT bag model; we just want to show that the droplet of quark matter under the periodic boundary condition inducing catalysis of chiral symmetry breaking and Nambu-Goldstone pions simultaneously is a reasonable result in the vacuum. In the future, it might be interesting to reinvestigate the bag model from the point of view of droplet-quark matter. 


\section{QUANTIZED FIRST-ORDER APPARENT PHASE TRANSITION IN COLD DROPLET QUARK MATTER}

In the last section, we showed that the ground state of the small system favors applying the PBC for quarks, and the zero-mode contribution becomes dominant in the small system. Now we investigate the chiral apparent phase transition at a high temperature and/or baryon density with changing of the finite size. For an infinite volume system, the NJL model predicts a critical end point for the chiral phase transition in the $\left(T, \mu_{B}\right)$ plane located at $\left(T^{E}=48 \mathrm{MeV}\right.$, $\left.\mu_{B}^{E}=994 \mathrm{MeV}\right)$ with $\mu_{B}=3 \mu_{q}$ the baryon chemical potential. When the size decreases from $L=\infty$ to $L=5 \mathrm{fm}$, which is much larger than the pion wavelength $\lambda_{\pi}$, there is almost no changing for the properties of the system.

When the size further decreases, it is found that the properties of the system start to change dramatically. As shown in Fig. 4, in a certain region of the chemical potential, there are two branches of first-order apparent phase transition (APT) showing up and two apparent critical end points (CEPs) appear on the $\left(T, \mu_{B}\right)$ plane for different sizes $L=5,4,3,2.5,2 \mathrm{fm}$, with the dashed lines corresponding to the branch of apparent phase transition at a lower chemical potential marked as APT1 and the solid lines corresponding to the branch of apparent phase transition at a larger chemical potential marked as APT2, respectively. The apparent CEPs corresponding to APT1 and APT2 are marked as "CEP1" and "CEP2" correspondingly. From Fig. 4, it is observed that, when the size decreases, CEP1 and CEP2 of these two branches have opposite behaviors: CEP2 moves to a higher chemical potential and lower temperature, and CEP1 moves to a lower chemical potential and higher temperature. When the size further decreases, APT2 and CEP2 disappear, and only APT1 and CEP1 show up. These results were also observed in Ref. [13].

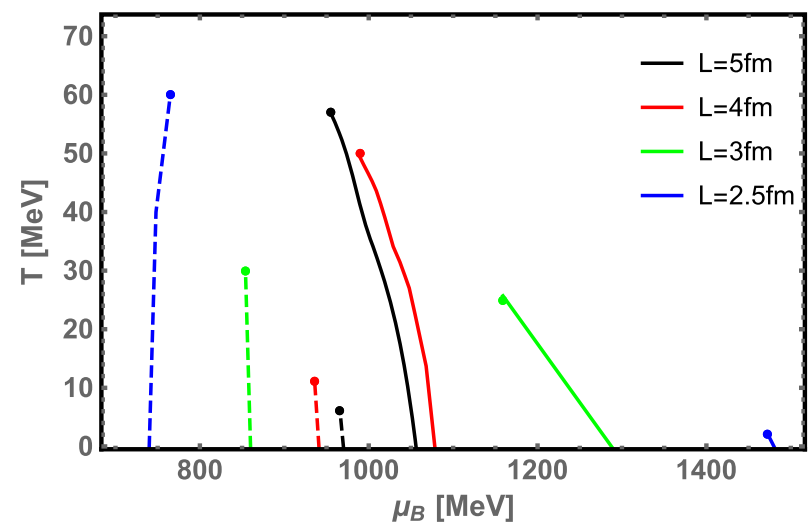

FIG. 4. Two branches of first-order apparent phase transitions and two sets of apparent critical end points in the $\left(T, \mu_{B}\right)$ plane for different sizes $L=5,4,3,2.5,2 \mathrm{fm}$. The dashed lines and solid lines correspond to APT1 and APT2, respectively.

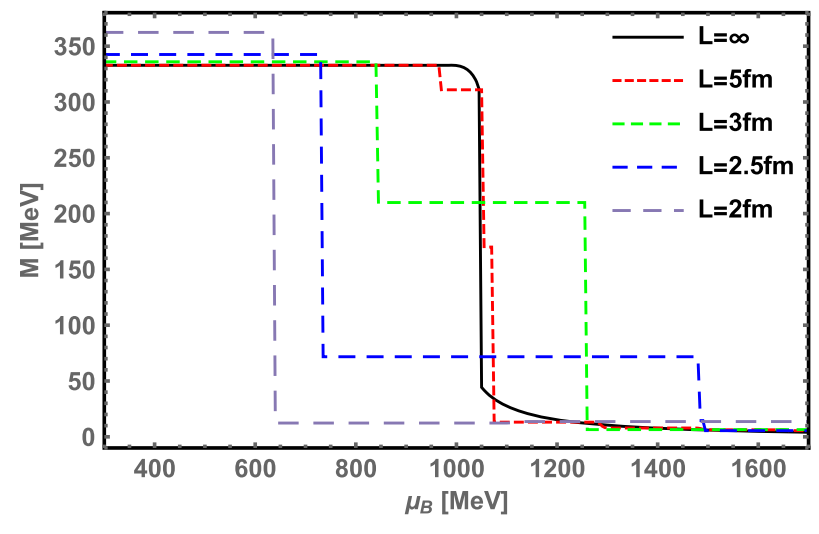

FIG. 5. The constituent quark mass as a function of the baryon chemical potential $\mu_{B}=3 \mu$ with different sizes of the system at zero temperature.

The showing up of two branches of first-order apparent phase transition can be clearly explained through Fig. 5, which shows the constituent quark mass as a function of the baryon chemical potential $\mu_{B}=3 \mu$ with different sizes of the system at zero temperature. In the region of $2 \mathrm{fm}<L<5 \mathrm{fm}$, the constituent quark mass jumps twice with the increase of the chemical potential. This multijump structure is caused by the zero-mode contribution. With the decrease of the system size, the zero-momentum contribution becomes more and more important. The first jump of the quark mass happens at a lower critical chemical potential and the magnitude of the jump becomes bigger and bigger, and the second jump of the quark mass appears at a higher chemical potential and its magnitude becomes smaller and smaller. Eventually, at a small enough size when the zero-mode contribution dominates, only the first jump shows up. Correspondingly, the location of the first CEP, i.e., CEP1, shifts to a lower critical chemical potential but a higher critical temperature, and the location of the second CEP, i.e., CEP2, moves to a higher and higher critical chemical potential but a lower critical temperature.

We call this multijump first-order apparent phase transition the quantized first-order apparent phase transition, where each first-order apparent phase transition is finished in several steps. We want to emphasize that such a quantized first-order apparent phase transition induced by quantized momentum is a brand-new quantum phenomena.

To show more clearly how these two branches of apparent phase transitions evolve with the system size. In Fig. 6, we show the 3-dimensional (3D) plot of the kurtosis of baryon number fluctuations $\kappa \sigma^{2}$ in the $\left(T, \mu_{B}\right)$ plane. The ratio of the fourth- to the second-order cumulant of quark number fluctuations $\kappa \sigma^{2}=\frac{c_{4}}{c_{2}}$ with $c_{n}=$ $V T^{3} \frac{\partial^{n}}{\partial(\mu / T)^{n}}\left(\frac{p}{T^{4}}\right)$ is used as a measurement to locate the apparent CEP in a beam-energy scan at the RHIC experiment [22]. At $L=\infty$, there is only one typical first-order phase boundary. It is observed clearly that, when the system 


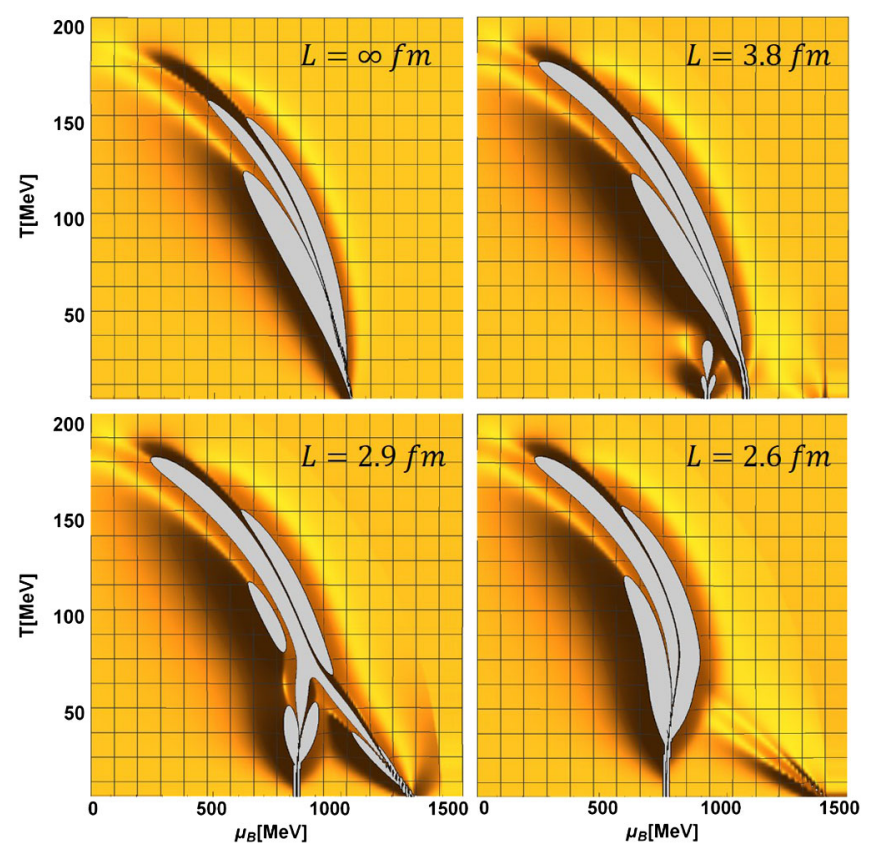

FIG. 6. The 3D plot of $\kappa \sigma^{2}$ in the $\left(T, \mu_{B}\right)$ plane with different sizes of the system.

size decreases, two branches of first-order apparent phase transition show up on the $\left(T, \mu_{B}\right)$ plane; one branch moves to a higher chemical potential and eventually disappears, and another branch shifts to a lower chemical potential region and then becomes dominant.

\section{ANALYZING THE APPEARANCE OF A QUANTIZED FIRST-ORDER APPARENT PHASE TRANSITION}

In the following, we analyze why the multijump structure of the first-order apparent phase transition can show up in certain small size systems, which was shown in our proceeding paper Ref. [23]. For this purpose, it can be much more transparent and simpler if we use the NJL model in the hard-cutoff regularization scheme, in which we can have the same qualitative results as that in the PauliVillars regularization scheme. By taking the hard-cutoff regularization for the momentum summation, the thermodynamical potential has the form of

$$
\begin{aligned}
\Omega_{\Lambda}= & \frac{\left(M-m_{0}\right)^{2}}{4 G}-\frac{2 N_{c} N_{f}}{V} \sum_{\vec{p}} \\
& \times\left\{E+T \ln \left(1+e^{-[(E+\mu) / T]}\right)+T \ln \left(1+e^{-[(E-\mu) / T]}\right)\right\} .
\end{aligned}
$$

Here the momentum should be smaller than the hard-cutoff $\Lambda: \Lambda^{2}>p^{2}=n\left(\frac{2 \pi}{L}\right)^{2}$.

In the following, we consider only the system at zero temperature $T=0$, and now the gap equation

$$
\frac{M-m}{2 G}=\frac{2 N_{c} N_{f}}{V} \sum_{\vec{p}} \frac{M}{E}[1-\theta(\mu-E)],
$$

where $\theta(x)$ is the step function. In the case of a small enough size so that $2 \pi / L>\Lambda$ with only the zero-momentum mode $n=0$ contributing to the momentum summation, the gap equation takes the form of

$$
\frac{M-m}{2 G}=\frac{2 N_{c} N_{f}}{V}\left[1-\theta\left(\mu-E_{0}\right)\right]
$$

and now $M=E_{0}=\sqrt{M^{2}+0(2 \pi / L)^{2}}$. The solution to this equation is simple and given below:

$$
M-m= \begin{cases}\frac{4 G N_{c} N_{f}}{V}, & \mu<\mu^{c}, \\ 0, & \mu>\mu^{c}\end{cases}
$$

with $\mu^{c}$ the critical quark chemical potential for the chiral apparent phase transition at zero temperature. Obviously, the above analysis is consistent with the line $L=2 \mathrm{fm}$ in Fig. 5.

Then we consider a little bit bigger size so that both the $n=0$ mode and $n=1$ mode can contribute to the momentum summation, and the gap equation takes the form of

$$
\begin{aligned}
\frac{M-m}{2 G}= & \frac{2 N_{c} N_{f}}{V}\left[1-\theta\left(\mu-E_{0}\right)\right] \\
& +6 \frac{2 N_{c} N_{f}}{V} \frac{M}{E_{1}}\left[1-\theta\left(\mu-E_{1}\right)\right],
\end{aligned}
$$

where $E_{1}=\sqrt{M^{2}+(2 \pi / L)^{2}}$. The second term on the right-hand side is from the contribution of the first mode, and 6 is the degeneracy number of the first mode. The solution to this equation is not as straightforward as Eq. (5) but can be calculated numerically.

We consider the chiral limit case with $m=0$. To show how the zero mode influences the constituent quark mass, in Fig. 7, we plot the constituent quark mass as a function of size $L$ at zero temperature and zero chemical potential, where $M_{0}$ and $M_{1}$ are obtained by solving gap equation (4) with and without a zero-mode contribution, respectively. It can be seen clearly that (i) at a large size when $L>5 \mathrm{fm}$, $M_{0}$ and $M_{1}$ are almost equivalent, which indicates that the contribution from the zero mode can be ignored when the size is large enough; (ii) when the size decreases and becomes smaller than $5 \mathrm{fm}, M_{0}$ and $M_{1}$ show completely opposite behaviors: $M_{0}$ rises quickly and goes to divergence with the decrease of the size, while $M_{1}$ decreases smoothly to zero at a very small size. In general, more step functions can appear in larger sizes; therefore, more jumps are expected. However, higher modes would not contribute a lot. For example, the second term on the right-hand side of Eq. (7) is quite small compared with the first term. It can 


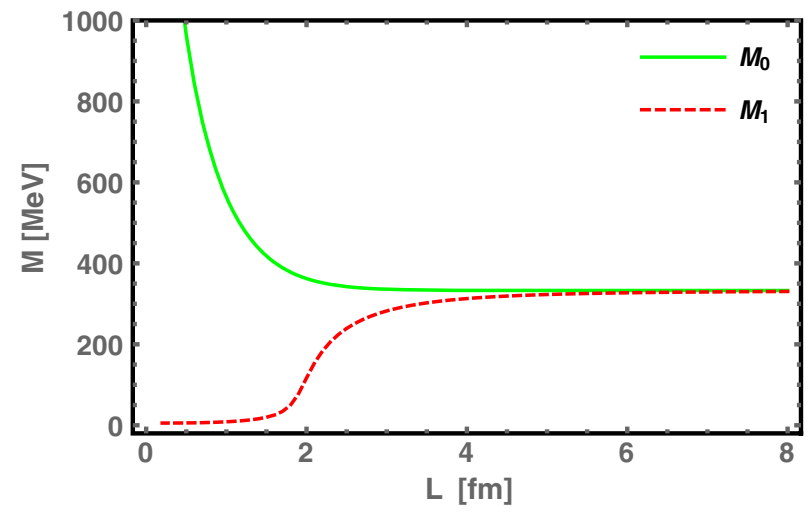

FIG. 7. The constituent quark mass $M_{0}$ with a zero-mode contribution and $M_{1}$ without zero-mode contributions as a function of the size $L$ at zero temperature and zero chemical potential. Here we take the chiral limit $m=0$.

be also verified from Fig. 5 that the magnitude of the second jump PT2 at $L=2.5 \mathrm{fm}$ is smaller than that at $L=3 \mathrm{fm}$.

With the help of $M_{0}$ and $M_{1}$, we can now understand the quantized first-order apparent phase transition in the size region of $2 \mathrm{fm}<L<5 \mathrm{fm}$. There are two apparent phase transitions, APT1 and APT2, as shown in Figs. 4 and 5, where APT1 can be understood as the jump between the chiral symmetry breaking phase with quark mass $M_{0}$ and the chiral partially "restoring" phase with quark mass $M_{1}$ and APT2 can be understood as the jump between the phase with quark mass $M_{1}$ and the chiral symmetry full restoration phase with $M=0$. At a large size when $L>5 \mathrm{fm}$, we can see that $M_{0}=M_{1}$, which indicates that the first branch phase transition PT1 vanishes. At a small size when $L<2 \mathrm{fm}$ where $M_{1}=0$, the second branch apparent phase transition APT2 vanishes. Therefore, there is only one jump of the first-order apparent phase transition at both cases with large size $L>5 \mathrm{fm}$ and small size $L<2 \mathrm{fm}$, but there appear two jumps for the first-order apparent phase transition in the region of $2 \mathrm{fm}<L<5 \mathrm{fm}$. This is exactly what we have obtained in Figs. 4 and 5.

From the above analysis, we can expect that the quantized first-order apparent phase transitions are common features for fermionic systems with a quantized momentum spectrum with a zero-mode contribution.

\section{CATALYSIS OF CHIRAL CONDENSATE AND QUANTIZED FIRST-ORDER PHASE TRANSITION UNDER A STRONG MAGNETIC FIELD}

Now consider a right-circular cylinder system with an infinite size along the $z$ axis but finite radius $r$ in the base plane perpendicular to the $z$ axis as shown in Fig. 8; then the momentum integral in the thermodynamic potential calculation is replaced by the momentum integral in the $z$ axis and momentum summation along the radius direction in the base plane:
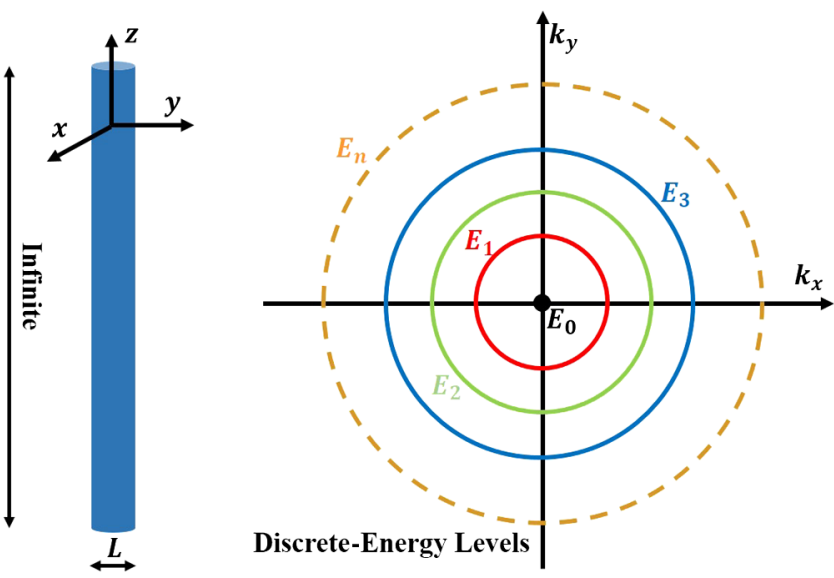

FIG. 8. The cylinder with a finite size at the $x-y$ plane and the discrete-energy levels.

$$
\begin{aligned}
\Omega= & \frac{\left(M-m_{0}\right)^{2}}{4 G}-\frac{2 N_{c} N_{f}}{S} \sum_{\vec{p}_{\perp}} \int_{-\infty}^{\infty} \frac{d p_{z}}{2 \pi} \\
& \times\left\{E+T \ln \left(1+e^{-[(E+\mu) / T]}\right)+T \ln \left(1+e^{-[(E-\mu) T]}\right)\right\},
\end{aligned}
$$

where $S$ is the area of the cylinder and $p_{\perp}$ the discrete momentum along the radius direction in the base plane. $p_{\perp}$ can be obtained by applying boundary conditions on the surface in the radius direction, for example, a boundary condition that requires a wave function to vanish on the surface. By applying different boundary conditions, different discrete momenta can be obtained and determine whether a zero-momentum mode is included. And, in the above equations, we sum all momenta in the base plane starting from $p_{\perp}=0$.

As we mentioned in the introduction, the catalysis of chiral symmetry breaking in a small system with a PBC including the zero-momentum-mode contribution is similar to the system of quark matter under strong magnetic field $B$. It is not difficult to understand the similarity between the two systems, if we recognize that, for a particle with charge $q$, the magnetic length $l$ is proportional to the inverse of the square root of the magnetic field, i.e., $l \sim \frac{1}{\sqrt{|q| B}}[19]$, which is similar to a right-circular cylinder system as shown in Fig. 8, and the stronger the magnetic field, the smaller the magnetic length will be. In the presence of a magnetic field, the momentum integral is replaced by a discrete momentum summation in the plane perpendicular to the magnetic field as shown in Fig. 8. Therefore, we expect that the quantized first-order phase transition can also show up in cold quark matter under a magnetic field.

The thermodynamic potential of quark matter in the presence of a magnetic field takes the form as given in Ref. [24]: 

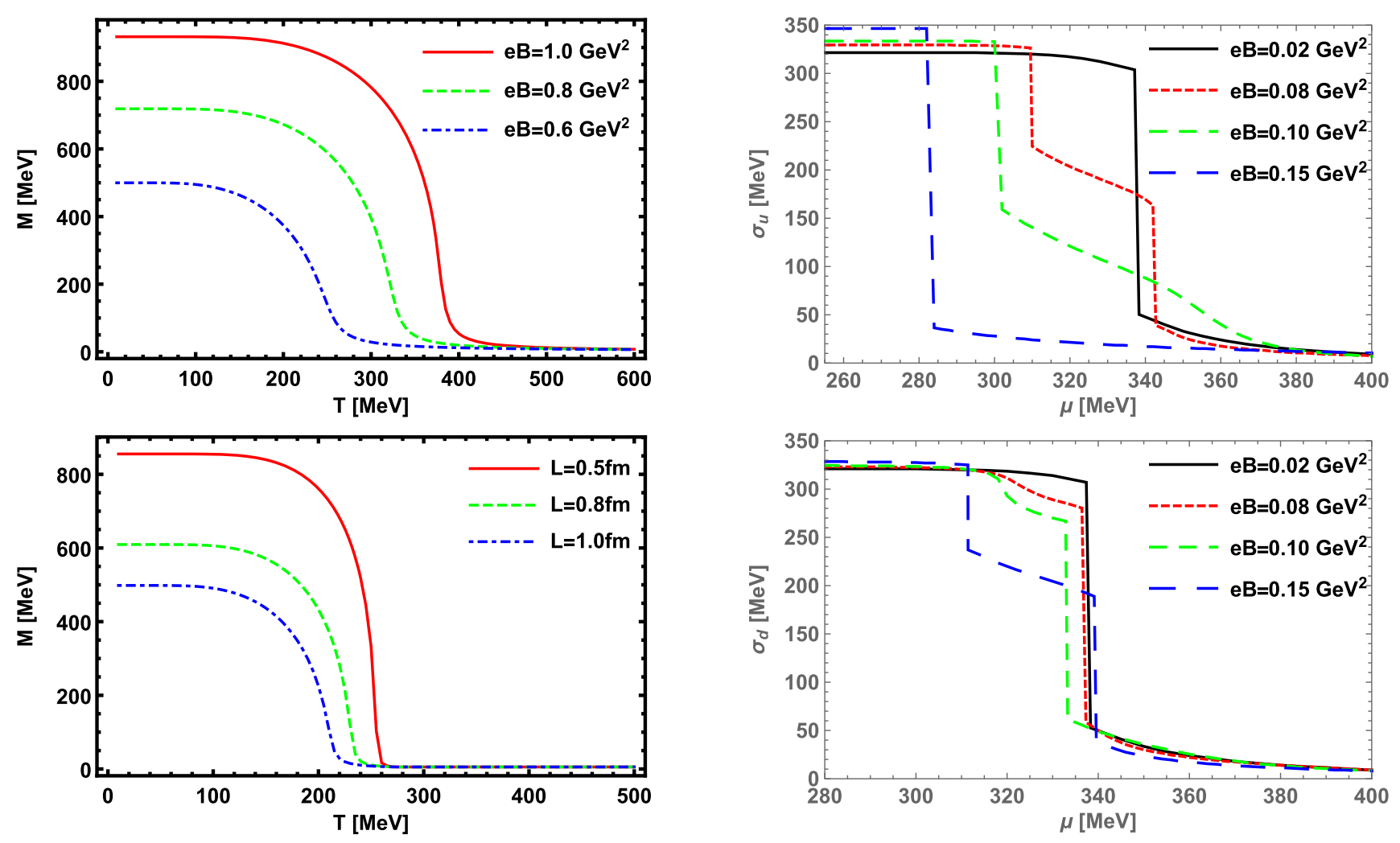

FIG. 9. Quark mass as a function of the temperature with only the lowest energy level taken into account under different magnitudes of the magnetic field (top) as well as for different radius $L$ for the cylinder (bottom).

$$
\begin{gathered}
\Omega=\frac{\sigma^{2}}{4 G}-N_{c} \sum_{f=u, d} \frac{\left|q_{f} B\right|}{2 \pi} \sum_{s, k} \alpha_{s, k} \int_{-\infty}^{\infty} \frac{d p_{z}}{2 \pi} \omega_{k}(p) \\
-T N_{c} \sum_{f=u, d} \frac{\left|q_{f} B\right|}{2 \pi} \sum_{s, k} \alpha_{s, k} \int_{-\infty}^{\infty} \frac{d p_{z}}{2 \pi} \\
{\left[\ln \left(1+e^{-\beta\left(\omega_{k}+\mu\right)}\right)+\ln \left(1+e^{-\beta\left(\omega_{k}-\mu\right)}\right)\right] .}
\end{gathered}
$$

Here $\omega_{k}=\sqrt{\left(\sigma+m_{0}\right)^{2}+p_{z}^{2}+2\left|q_{f} B\right| k}$ is the dispersion relationship and $k=0,1,2, \ldots$ a non-negative integer number labeling the Landau levels, and the spin degeneracy factor $\alpha_{s, k}$ is given below:

$$
\alpha_{s, k}=\left\{\begin{array}{lll}
\delta_{s,+1} & \text { for } k=0, & q B>0 \\
\delta_{s,-1} & \text { for } k=0, & q B<0 \\
1 & \text { for } k \neq 0, &
\end{array}\right.
$$

with $s= \pm$ the spin factor. Here we take the magnetic field $B$ along the $z$ axis and consider only $u$ and $d$ quarks. Similar to the case without a magnetic field, the ground state of the system can be determined by solving the gap equation $\partial \Omega / \partial \sigma=0$.

The quark mass as a function of the temperature under different magnitudes of a strong magnetic field is shown in

FIG. 10. Chiral condensates for the $u$ quark (upper figure) and $d$ quark (lower figure) as a function of the chemical potential for different magnitudes of the magnetic field.

the upper figure in Fig. 9. It is observed that both the quark mass and the corresponding critical temperature increase as the magnetic field increases, which is known as magnetic catalysis $[17,18]$. Similarly, the catalysis of the chiral condensate is also shown in the cylinder system as the size decreases shown in the lower figure in Fig. 9. The small size plays a similar role as the strong magnetic field, and they both enhance chiral symmetry breaking. The stronger the magnetic field and the smaller the size is, the more contribution from the zero mode.

Now we consider cold dense quark matter under a strong magnetic field. Because the $u$ quark and $d$ quark carry different electric charges, the Fermi surfaces for $u$ and $d$ quarks are splitting, and their critical chemical potentials for chiral phase transitions are also different. The chiral condensate at zero temperature for $u, d$ quarks at different magnitudes of the magnetic field are shown in Fig. 10. As we expected, there are also quantized first-order chiral phase transitions showing up for the $u$ quark and $d$ quark. To our knowledge, the quantized first-order chiral phase transition phenomena under a strong magnetic field has not yet been observed in other literature except Ref. [25]. It is worth mentioning that, in the case of a strong magnetic field, the thermodynamic limit of infinite volume can be taken. Hence, the phase transition under a strong magnetic field in this section is an actual phase transition which is 
different from the apparent phase transition that happens in a finite size system.

\section{SUMMARY AND DISCUSSION}

Even though the finite size effect in QCD physics has attracted lots of interest for more than three decades, normally, both the periodic and the antiperiodic spatial boundary condition are applied for fermions. In this work, we find that, if there is no other physical constraint, the ground state of quark matter favors the periodic spatial boundary condition, in which the zero-momentum mode is taken into account. In this stable small system, the catalysis of chiral symmetry breaking is observed with the decrease of the system size, while the pions excited from the droplet vacuum remain as pseudo-Nambu-Goldstone bosons. The catalysis of chiral symmetry breaking and pseudo-NambuGoldstone pions in a small system are similar to those in quark matter under strong magnetic fields. The similarity between these two systems is understandable, because the stronger the magnetic field, the smaller the magnetic length of the charged particle will be.

For the periodic boundary condition, one can obtain the catalysis of chiral symmetry breaking in the vacuum at a small size, which indicates that the constituent quark becomes heavier and its wavelength becomes much smaller. We can imagine that the droplet-quark matter with a periodic condition is a bag with dynamical massive quarks inside the bag surrounded by a light pseudoNambu-Goldstone pions' cloud.

Furthermore, it is found that the zero-momentum-mode contribution brings significant change of the chiral apparent phase transition in a droplet of cold dense quark matter: The first-order chiral apparent phase transition becomes quantized, which is a brand-new quantized phenomena. We want to emphasize that this is the first time to observe the quantized first-order apparent phase transition in the literature. As we analyzed in this work, the quantized first-order phase transition is induced by the quantized momentum, and in this case the zero-momentum-mode contribution becomes non-negligible. It is expected that such a quantized first-order apparent phase transition is a common feature for a system with quantized momentum. Therefore, we also observe such quantized first-order phase transition phenomena in quark matter under a strong magnetic field, and we expect such phenomena will also show up in some small systems in condensed matter.

Last, we expect that this new phenomena will largely affect the equation of state with small droplet quark matter inside a neutron star and, thus, affect the radius-mass relation of the neutron star. When considering the mixed phase of quark matter and nuclear matter, one has to solve the Wigner-Seitz cell structure, e.g., drop (bubble), rod (tube), and slab structure, and the size of the Wigner-Seitz cell can be as small as several femtometers as shown in Ref. [16]. For the small size of the Wigner-Seitz cell in neutron stars, the work of investigating how the zero-mode contribution will affect the equation of state and, thus, the neutron star properties is in progress [26]. Also, it is noticed that, in the future, it is worthy to perform calculations beyond the mean-field approximation.

\section{ACKNOWLEDGMENTS}

We thank T. Hatsuda and R. Pisarski for valuable discussions. This work is supported in part by the NSFC under Grants No. 11725523, No. 11735007, and No. 11261130311 (CRC 110 by DFG and NSFC), Chinese Academy of Sciences under Grant No. XDPB09, and the start-up funding from University of Chinese Academy of Sciences (UCAS).
[1] H. K. Khattak, P. Bianucci, and A. D. Slepkov, Proc. Natl. Acad. Sci. U.S.A. 116, 4000 (2019).

[2] M. E. Fisher and M. N. Barber, Phys. Rev. Lett. 28, 1516 (1972).

[3] M. N. Barber, Finite-size scaling, in Phase Transitions and Critical Phenomena, edited by C. Domb and J. L. Lebowitz (Academic Press, London, 1983), Vol. 8.

[4] E. Brezin and J. Zinn-Justin, Nucl. Phys. B257, 867 (1985).

[5] M. Luscher, Commun. Math. Phys. 104, 177 (1986); 105, 153 (1986).

[6] J. Gasser and H. Leutwyler, Nucl. Phys. B307, 763 (1988).

[7] J. Gasser and H. Leutwyler, Phys. Lett. B 188, 477 (1987).

[8] O. Kiriyama, T. Kodama, and T. Koide, arXiv:hep-ph/ 0602086.
[9] G. y. Shao, L. Chang, Y. x. Liu, and X. 1. Wang, Phys. Rev. D 73, 076003 (2006).

[10] L. F. Palhares, E. S. Fraga, and T. Kodama, J. Phys. G 38, 085101 (2011).

[11] J. Braun, B. Klein, H.-J. Pirner, and A. H. Rezaeian, Phys. Rev. D 73, 074010 (2006); J. Braun, B. Klein, and H. J. Pirner, Phys. Rev. D 72, 034017 (2005); J. Braun, B. Klein, and B. J. Schaefer, Phys. Lett. B 713, 216 (2012).

[12] B. L. Li, Z. F. Cui, B. W. Zhou, S. An, L. P. Zhang, and H. S. Zong, Nucl. Phys. B938, 298 (2019); Q. W. Wang, Y. Xia, C. Shi, and H. S. Zong, arXiv:1802.00258; Y. P. Zhao, P. L. Yin, Z. H. Yu, and H. S. Zong, Nucl. Phys. B952, 114919 (2020).

[13] G. Almasi, R. Pisarski, and V. Skokov, Phys. Rev. D 95, 056015 (2017). 
[14] B. Klein, Phys. Rep. 707-708, 1 (2017).

[15] H. Heiselberg, C. J. Pethick, and E. F. Staubo, Phys. Rev. Lett. 70, 1355 (1993).

[16] X. Wu and H. Shen, Phys. Rev. C 96, 025802 (2017); 99, 065802 (2019).

[17] V. P. Gusynin, V. A. Miransky, and I. A. Shovkovy, Phys. Rev. Lett. 73, 3499 (1994); 76, 1005(E) (1996).

[18] V. A. Miransky and I. A. Shovkovy, Phys. Rep. 576, 1 (2015).

[19] D. Tong, arXiv:1606.06687.

[20] S. P. Klevansky, Rev. Mod. Phys. 64, 649 (1992).
[21] A. Chodos, R. L. Jaffe, K. Johnson, C. B. Thorn, and V. F. Weisskopf, Phys. Rev. D 9, 3471 (1974).

[22] X. Luo and N. Xu, Nucl. Sci. Tech. 28, 112 (2017).

[23] K. Xu and M. Huang, Proc. Sci., CORFU2018 (2019) 172 [arXiv:1904.01154].

[24] J. Chao, P. Chu, and M. Huang, Phys. Rev. D 88, 054009 (2013).

[25] P. Costa, M. Ferreira, H. Hansen, D. P. Menezes, and C. Providncia, Phys. Rev. D 89, 056013 (2014).

[26] K. Xu and M. Huang (to be published). 